Research Article

\title{
An Overview of Technological Revolution in Satellite Image Analysis
}

\author{
Jenice Aroma R* and Kumudha Raimond
}

Department of Computer Science \& Engineering, Karunya University, Coimbatore, India

Received 1 July 2016; Accepted 31 August 2016

\begin{abstract}
The satellite image based applications are highly utilized nowadays from simple purposes like vehicle navigation to complex surveillance and virtual environment modeling projects. On increased population rate, the depletion of natural resources is highly unavoidable and it leads to increased threats on natural hazards. In order to protect and overcome the physical losses on devastation of properties, the risk mapping models such as weather forecasts, drought modeling and other hazard assessment models are in need. Though many intelligent risk mapping models do exist, the high level of intelligence needed to predict the real time events is still unresolved. And it leads to increased research efforts on designing intelligent assessment models using more advanced machine learning (ML) schemes for better satellite image interpretation. This paper focuses on bringing a clear understanding on satellite image interpretation methods right from the traditional statistical models to the more recent ML methods through stating the gradual revolution in satellite image analysis.
\end{abstract}

Keywords: Satellite image, Remote Sensing, Machine Learning, Statistical methods.

\section{Introduction}

The remote sensing is a field of acquiring the information of an object through sensing either own or artificially emitted electromagnetic radiation. It can be either passive or active means of remote sensing. In passive mode of remote sensing, the sensors detect naturally available energy of an object due to sunlight and other infrared radiations of the electromagnetic spectrum reflected from the earth surface as in Fig.1.(a). In active remote sensing, the sensors generate an artificial radiation to monitor an object on the earth surface as in Fig.1.(b). These observed signals are then digitized as satellite images in the ground stations. The passive sensors are limited on night sensing due to lack of sunlight. But active sensors are more advantageous which can be acquired all through the day and even under heavy cloud and fog conditions [1].
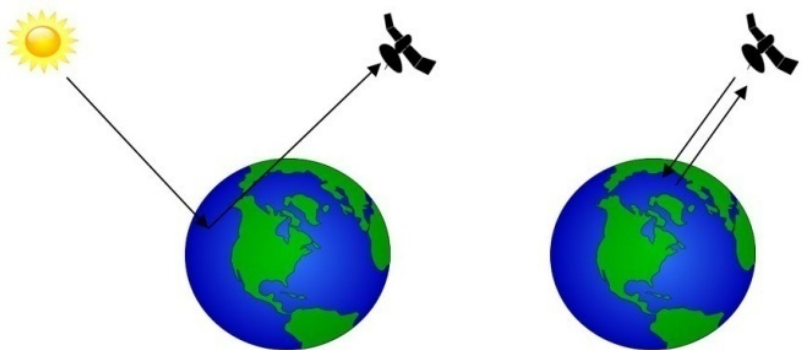

Fig.1 (a) Passive Remote Sensing ; (b) Active Remote Sensing.

The Satellite images are widely used for better visualization of distant objects, weather prediction models and other spatial object recognition models. As the field of

*E-mail address: jenicearoma@gmail.com

ISSN: 1791-2377 @ 2016 Eastern Macedonia and Thrace Institute of Technology. All rights reserved. remote sensing is highly associated with observing the real life events, the human intelligence based ML methods can be of great benefit in discriminating or monitoring the dayto-day life on earth.

The satellite sensor variations such as spatial, spectral and radiometric resolution along with change of viewpoint bring huge diversities into satellite image representations [2]. The fusion techniques can integrate images of the above said different variations and could enrich the information available of the chosen study area. Le Yu et al., in 2008 performed a completely automatic registration in multi sensor satellite images using point features [3]. Barbara Zitova et al, in 2003 made an extensive survey on available image registration methods [4]. But in case of large areas, it is still a laborious task to apply image fusion.

The conventional satellite image models are mostly based on statistical methods like maximum likelihood approach, Principal Component Analysis (PCA), Linear Discriminant Analysis (LDA) and other regression based models etc-. Most of these traditional algorithms suffer with lack of logical reasoning.

By incorporating human level reasoning into these traditional models, a vibrant progress has been achieved in ML methods. But supervised ML methods suffer with high computational complexity due to tedious training processes and insufficient ground truth data for labeling. In order to overcome these issues, the training phase must be limited which can be achieved through unsupervised mode of learning. Further, the accuracy of classification either in supervised or unsupervised can be improved using hybridization. Similarly, parallel processing of data which could improve the issues on speed of computation through higher level Graphical Processing Unit (GPU) architectures is in progress [5].

The most encouraging factor of satellite image analysis is the involvement of different domain experts and it has 
been portrayed in Section 2. The extensive applications based on statistical and ML methods to exploit satellite images are detailed in the Sections 3 and 4 respectively.

\section{Multiple domain experts in Satellite image analysis}

The satellite images are utilized by different users such as researchers, students and government agencies. However, the majority of users remain with either remote sensing or ML community with respect to few factors like application outcome, research focus, image analysis and type of tools.

\subsection{Remote Sensing Experts}

The Remote sensing community includes geologists, earth scientists, climate scientists and archaeologists etc- who relies more on the field study than ML community. They use the ground measurements from sensors installed in the field of study for validation of models.

The use of Geographical Information System (GIS) tools which is highly compatible for exploiting the necessary information from satellite images with built-in functions for complex level of processing is also more prevalent among this community. J.S.Rawat et al. (2013) performed a supervised classification for detecting land cover change in the chosen study area using maximum likelihood approach in Erdas Imagine [6].

Fei Yuan et al. (2005) performed initial land cover classification using guided clustering and maximum likelihood approach. And post classification to resolve the errors is carried out using a rule-based spatial model in Erdas Imagine [7]. Michael A. DeVasto et al. (2012) performed automated grain boundary detection in satellite images using ArcGIS [8]. Tapas R. Martha et al., in 2014 performed visual analysis of change estimation in pan sharpened very high resolution images for detecting damage estimation using Erdas Imagine [9]. The use of derived indices along with geospatial tools analysis is also more popular among this research community in tracing or predicting the environmental changes. The combination of different vegetation indices could achieve a better prediction. Usually, the meteorological drought occurs a few months earlier than agricultural drought and use of single index is a serious limitation in drought monitoring. Hence integration of multiple indices is a good choice [10].

Zhang Rongqun (2011) proposed a knowledge rule based approach with integrated spectral and spatial features to deal with the limits on spectral information based classification since the spectral information is insufficient to discriminate the objects with similar spectra. [11].

\subsection{Machine Learning Experts}

The scope of research among ML community emerges with resolving the limitations on statistical models experienced by the remote sensing researchers through enhancing the level of reasoning. The following are the major factors that are considered for resolving the issues on traditional satellite image analysis:

- To enrich the reasoning capability of models

- To handle huge dimensional data processing in satellite image analysis.

- To overcome the limitations of traditional ML classifiers through hybridization or object based classification
The satellite images are used in climate based models for monitoring seasons and also hazards like weather forecast, agricultural drought prediction, rainfall prediction, flood mapping etc.- [12]. The recent innovations in satellite sensor instruments lead to the availability of wide range of satellite images with more increased resolution and dimensionality. Hence, more advanced crop health tracking applications, road network detection, vehicle navigation and urban planning models for cities etc- has also become beneficial in aiding humanity $[13,14,15,16]$.

P. Sathya et al. (2011) compared the performance of Kmeans clustering and back-propagation neural network for effective image segmentation and classification in satellite images using dense count values of the pixel intensities [17]. But it would be highly advantageous to use more advanced intelligence schemes like Deep Neural Network (DNN) in satellite image analysis which could bring out improved level of reasoning with reduced training complexities.

After 2006, the deep learning schemes are profound in handling complex computations of higher level abstractions [18] and are moving beyond the capacity of shallow machine learning era. The complexities on training the deep networks with huge number of hidden layers are resolved to a greater extent by a greedy learning based algorithm [19]. These DNN learning schemes are more vibrant now, in the field of speech, natural language processing and internet technologies [20].

The following sections 3 and 4 portray the works of both the research communities to exploit the level of intelligence through statistical and ML based methods for satellite image interpretation models.

\section{Statistical Methods}

The conventional spatial models are based on statistical methods, which are highly dependent on spectral only information. It can achieve better accuracy but the lack of reasoning leads to reduced prediction rate than machine learning models.

D.I.F Grimes et al. (1999) merged the estimates of ground and satellite derived rainfall observations of same region through calculating a weighted average of both the satellite and guage values for each satellite pixel [21]. Farid Melgani et al. (2002) made an attempt to fuse spectral information with spatial and temporal information using Markov Random Fields (MRF). The results showed improved classification accuracy with the increased level of contextual information [22].

Susan Malaso, (2015) has developed a Frost risk mapping model using pixel based classification of satellite images in manipulating the minimum temperature [23]. It is a highly evident mode of satellite image interpretation where the extracted temperature values are analysed for tracing the previous change outcome or for future prediction.

In order to estimate the temperature from brightness values of the satellite image pixels, the proper methods for extraction of spectral reflectance is must. The Landsat 7 Science Data User's Handbook procedure can be followed to estimate the temperature from Landsat data. The following Tab. 1 illustrates few works from the literature that applies statistical methods for satellite image interpretation.

The previous studies and works on statistical image classification methods clearly reveal that improving the existing statistical based image classification algorithms alone is insufficient to achieve high level of accuracy. 
Hence, it is profound to step up for new schemes in image classification using the recent advances in ML.

Table. 1. Statistical Models for Satellite Image Analysis

\begin{tabular}{|c|c|c|}
\hline Author & Method & Advantage \\
\hline $\begin{array}{l}\text { Liu Haijiang et al., } \\
2007 \text { [24] }\end{array}$ & $\begin{array}{l}\text { Monitoring the Land } \\
\text { cover change for } \\
\text { desertification of an } \\
\text { island using Maximum } \\
\text { Likelihood Classifier. }\end{array}$ & $\begin{array}{l}\text { Simple mode of } \\
\text { classification. }\end{array}$ \\
\hline $\begin{array}{l}\text { H.S.Lim et al., } 2007 \\
\text { [25] }\end{array}$ & $\begin{array}{l}\text { Air pollution } \\
\text { monitoring using } \\
\text { PM10 values extracted } \\
\text { from Landsat TM and } \\
\text { Aerosol Optical } \\
\text { Thickness (AOT) } \\
\text { values from gauges. }\end{array}$ & $\begin{array}{l}\text { Integration of } \\
\text { satellite derived and } \\
\text { in-situ data. }\end{array}$ \\
\hline $\begin{array}{l}\text { J.Tian et al., } 2007 \\
\text { [26]. }\end{array}$ & $\begin{array}{l}\text { Studied the importance } \\
\text { of validating the } \\
\text { correlation of satellite } \\
\text { derived and gauge } \\
\text { measurements }\end{array}$ & $\begin{array}{l}\text { Experiments are } \\
\text { analyzed with } \\
\text { different seasons. }\end{array}$ \\
\hline $\begin{array}{l}\text { Francis Padula et } \\
\text { al., 2010 [27]. }\end{array}$ & $\begin{array}{l}\text { Land cover change } \\
\text { detection using PCA. }\end{array}$ & $\begin{array}{l}\text { Fused Landsat and } \\
\text { MODIS data is used } \\
\text { for better data } \\
\text { resolution }\end{array}$ \\
\hline $\begin{array}{l}\text { Brian P. Salmon et } \\
\text { al., } 2011 \text { [28]. }\end{array}$ & $\begin{array}{l}\text { Unsupervised Land } \\
\text { cover change detection } \\
\text { in MODIS data using } \\
\text { sequential time series } \\
\text { analysis. }\end{array}$ & $\begin{array}{l}\text { Lesser training time } \\
\text { than supervised } \\
\text { learning methods. }\end{array}$ \\
\hline $\begin{array}{l}\text { Deepti Sharma et } \\
\text { al., 2011 [29]. }\end{array}$ & $\begin{array}{l}\text { Studied dust storm } \\
\text { effects using aerosol } \\
\text { products acquired from } \\
\text { both satellite derived } \\
\text { and ground } \\
\text { measurements. }\end{array}$ & $\begin{array}{l}\text { Both ground based } \\
\text { and atmospheric } \\
\text { parameters } \\
\text { considered. }\end{array}$ \\
\hline $\begin{array}{l}\text { Biswadip Gharai et } \\
\text { al., } 2013 \text { [30]. }\end{array}$ & $\begin{array}{l}\text { Fusion of MODIS and } \\
\text { CALIPSO (LIDAR) } \\
\text { Data to study the Dust } \\
\text { storm effects. }\end{array}$ & $\begin{array}{l}\text { Use of LIDAR data } \\
\text { favors studying the } \\
\text { vertical uplift of dust } \\
\text { in air. }\end{array}$ \\
\hline
\end{tabular}

\section{Machine Learning Methods}

The statistical models are not efficient in prediction due to the irregularly varying patterns of voluminous data which can never be deduced from a formula without reasoning capability. Thus, ML techniques are found to be good in deriving prediction models as it learns through experience during training phase [31].

The role of Artificial Neural Network (ANN) begins by 1990 overcoming the issues of statistical pattern recognition methods like Bayesian classifiers and Parzen windows [32].In general, the supervised learning methods are highly limited by rigorous training time which leads to longer computation time. To reduce this time complexity, the training phase must be limited which can be achieved through unsupervised mode of learning.

Further, the improvement of advanced ML classifiers are carried out in two directions, (i) Hybridization and (ii) Object Based Image Analysis (OBIA) which are detailed in the following sections.

\subsection{Hybrid methods}

The hybridization of different machine learning methods and the integration of statistical and machine learning methods could acquire more efficient results. Giorgio Giacinto et al., (2000) proposed an approach with combination of neural and statistical algorithms in a simple design phase to clearly reveal that every algorithm is significant in solving a specific issue and no single algorithm is proved to be perfect [33].

.Castellana et al., (2007) performed the combination of both supervised and unsupervised mode of change detection on pixel based method to achieve better classification of remote sensing images [34]. Pabitra Mitra et al., (2004) combined active learning method with Support Vector Machine (SVM) and developed an active SVM with reduced labeled data for classification [35].

Qihao Weng et al., (2004) combined Maximum likelihood and Decision Tree classifier to estimate the vegetation abundance in a land cover considering the Land Surface Temperature (LST) and vegetation relationship [36]. Few more hybrid models for satellite image interpretation have been tabulated below in the following Tab. 2 .

Table. 2. Hybrid ML Approaches for Satellite Image Analysis

\begin{tabular}{|c|c|c|}
\hline Author & Method & Advantage \\
\hline $\begin{array}{l}\text { Rubia et al., } 2009 \\
{[37] .}\end{array}$ & $\begin{array}{lr}\text { Combined } & \text { Fuzzy } \\
\text { Inference } & \text { System } \\
\text { (FIS) and } & \text { Genetic } \\
\text { Algorithm (GA) }\end{array}$ & $\begin{array}{l}\text { Both spatial and } \\
\text { temporal analysis } \\
\text { over forest land } \\
\text { cover. }\end{array}$ \\
\hline $\begin{array}{l}\text { Zhiding Yu et al., } \\
2010 \text { [38]. }\end{array}$ & $\begin{array}{l}\text { Combined Ant } \\
\text { colony algorithm } \\
\text { and FCM }\end{array}$ & $\begin{array}{l}\text { Improved FCM's } \\
\text { limit on sensitive } \\
\text { to noisy data and } \\
\text { random } \\
\text { initialization of } \\
\text { parameters. }\end{array}$ \\
\hline $\begin{array}{l}\text { Mohammad } \\
\text { Awad et al., } 2010 \\
\text { [39]. }\end{array}$ & $\begin{array}{l}\text { Combined different } \\
\text { threshold functions } \\
\text { with Traditional } \\
\text { Self Organizing } \\
\text { Maps (SOM) }\end{array}$ & $\begin{array}{l}\text { Overcome the } \\
\text { under and over } \\
\text { segmentation } \\
\text { issues of SOM. }\end{array}$ \\
\hline $\begin{array}{l}\text { Ashish Ghosh et } \\
\text { al., } 2011 \text { [40]. }\end{array}$ & $\begin{array}{l}\text { Performed } \\
\text { unsupervised } \\
\text { clustering and then } \\
\text { combined with GA } \\
\text { and Simulated } \\
\text { annealing. }\end{array}$ & $\begin{array}{l}\text { Improved random } \\
\text { initialization } \\
\text { issues on } \\
\text { clustering }\end{array}$ \\
\hline $\begin{array}{l}\text { Xiang Yang } \\
\text { Wang et al., } 2012 \\
{[41] .}\end{array}$ & $\begin{array}{l}\text { Combined } \\
\text { and SVM }\end{array}$ & $\begin{array}{lr}\text { Increased } & \\
\text { segmentation } & \\
\text { quality } & \text { and } \\
\text { reduced } & \text { time } \\
\text { complexity. } & \end{array}$ \\
\hline
\end{tabular}

Kazim Hanbay et al., (2014) have proposed a novel image segmentation algorithm for Synthetic Aperture Radar images using the combination of improved Artificial Bee Colony (ABC) algorithm and Neutrosophic set. Here, the hybrid feature extraction model using neutrosophic and cooccurrence matrices achieved effective localization of edges in images [42]. Similarly, A.K.Bhandari et al., (2015) proposed a Modified ABC (MABC) algorithm for satellite image segmentation with very less control parameters and on applying multi-level thresholding functions like Kapur's, Otsu and Tsallis objective function [43].The Genetic Algorithm (GA) is used in automatic feature selection methods since it is well known for high level of optimization in refining the irrelevant and noisy features. The fuzzy algorithms stand high in handling the level of uncertainty. Hence, the fusion of these approaches could enhance the level of classification accuracy in ML models.

Other than this, novel ideas from natural heuristics, human anatomy and molecular reactions are also imparted in evolutionary algorithms for improving the performance of existing ML methods. The limitations of traditional clustering algorithms on slower convergence speed and effects on wrong initialization parameters are resolved using the Quantum inspired Multi-objective Evolutionary 
Clustering (QMEC) algorithm which is based on the quantum principle of energy interactions [44].

\subsection{Object based Image Analysis}

The rate of interest in OBIA based methods increased after 2006. The OBIA methods depend on both the spatial and spectral information of images through considering texture, pixel proximity and geometric attributes of image objects.

Jose M. Pena, (2014), mentioned that conventional ML methods are generally based on spectral information but the recent classifiers are mostly object level which considers both the spectral and spatial features [45]. T. Blaschke, (2010) stated that use of OBIA is more prevalent than perpixel methods and Image segmentation is the major building block of OBIA [46]. Hay et al., (2005) performed a higher level of classification using segmented objects to classify the forestland cover into individual tree crowns [47].

Muhammad Shahbaz et al., (2012), performed object based image classification using decision trees and achieved higher degree of robustness and accuracy for object recognition [48]. Rutherford V. Platt, (2008) stated that their proposed approach on OBIA and the performance is superior than per-pixel method for mixed urban and agro land cover classification [49]. Petra Helmholz et al., (2014) performed semi-supervised classification in high-resolution satellite images through the combination of both pixel and object based classification. Initially, pixel based classification is done using Markov Random Field to extract agricultural regions. Later these extracted regions are classified using SVM [50].

Turker et al., (2008) proposed an approach to detect damaged building in an earthquake in Turkey by 1999 using OBIA. The post event aerial images are applied with watershed segmentation to be classified as damaged or undamaged [51]. Khudairy et al., (2005) used the pre-war images acquired from IKONOS for both Jenin and Brest of palestenian territory. The mathematical morphological operators are applied for automated identification of damaged areas. The results are more superior in OBIA than per-pixel approach [52]. L. Monika Moskal et al., (2011) analyzed the aerial datasets using OBIA for tree cover assessments. The results achieved are compared with that of pixel based analysis and OBIA is found to be superior [53].

Normally, in moderate resolution images every individual pixel may cover large spatial region and this increased data resolution may lead to challenges while using traditional approaches. Hence, OBIA is found to be more prominent that includes the use of both spectral \& spatial data, which is limited in per-pixel analysis of spectral information.

\section{Conclusion}

The recent trends in the image analysis schemes are a great boon to the advancements of satellite image applications in all sorts of fields. The importance of satellite image properties, different sorts of research in progress and other widely used methods are briefed for mapping the revolutionary growth of satellite image analysis. The general fact inferred from this study reveals that the need for efficient real time applications using satellite images is very high and it can come true by exploring the capabilities of unsupervised learning methods, considering the human cognition schemes and the improvements on effective hybridization. The future works of this study can be extended through exploring the available nature inspired algorithms for improved hybridization schemes and more advanced machine learning methods to design an intelligent model for satellite image analysis.

\section{References}

1. Shefali Aggarwal, Principles of Remote Sensing, Satellite Remote Sensing and GIS Applications in Agricultural Meteorology, Proceedings of the Training workshop, pp. 23-38, (2003).

2. Jenice Aroma $\mathrm{R}$ and Kumudha Raimond, A Review on availability of Remote Sensing Data, IEEE International conference on Technological Innovation in ICT for Agriculture and Rural Development, pp. 200-205, (2015).

3. Le Yu, Dengrong Zhang and Eun-Jung Holden, A fast and fully automatic registration approach based on point features for multisource remote sensing images, Computers \& Geosciences, 34, pp. 838-848, (2008).

4. Barbara Zitova and Jan Flusser, Image Registration methods: a survey, Image and Vision Computing, 21, pp. 977-1000, (2003).

5. Sergio Bernabe, Antonio Plaza, Prashanth Reddy Marpu, Jon Atli Benediktsson, A new parallel tool for classification of remotely sensed imagery, Computers \& Geosciences, 46, pp. 208-218, (2012).

6. J.S.Rawat, Vivekanand Biswas and Manish Kumar, Changes in land use/cover using geospatial techniques: A case study of Ramnagar town area, district Nainital, Uttarakhand, India, The Egyptian Journal of Remote Sensing and Space Sciences, 16, pp. 111-117, (2013).

7. Fei Yuan, Kali E. Sawaya, Brian C. Loeffelholz, Marvin E. Bauer, Land cover classification and change analysis of the Twin Cities (Minnesota) Metropolitan Area by multitemporal Landsat remote sensing, Remote Sensing of Environment, 98, pp. 317-328, (2005).

8. Michael A. DeVasto, Dyanna M. Czeck, Prajukti Bhattacharyya, Using image analysis and ArcGIS to improve automatic grain boundary detection and quantify geological images, Computers \& Geosciences, pp. 38-45, (2012).
9. Tapas R. Martha, K.Babu Govindharaj, K.Vinod Kumar, Damage and geological assessment of the 18 September $2011 \mathrm{mw} 6.9$ Earthquake in Sikkim, India using very high resolution satellite data, Geoscience Frontiers, pp. 1-14, (2014).

10. Zengchao Hao and Amir Aghakouchak, A Nonparametric Multivariate Multi-Index Drought Monitoring Framework, Journal of Hydrometeorology, 15, 89-101, (2014).

11. Zhang Rongqun and Zhu Daolin, Study of land cover classification based on knowledge rules using high-resolution remote sensing images, Expert Systems with Applications, 38, pp. 3647-3652, (2011).

12. P.S Roy, R.S.Dwivedi and D.Vijayan, Remote Sensing Applications , National Remote Sensing Centre, Technical Document, (2010).

13. Saptarshi Mondal, C.Jeganathan, Nithish Kumar Sinha, Harshit Rajan, Tanmoy Roy and Praveen Kumar, Extracting seasonal cropping patterns using multi-temporal vegetation indices from IRS-LISS III Data in Muzaffarpur District of Bihar, India, 17,pp. 123-134, (2014).

14. Jiaping Zhao and Suya You, "Road Network Extraction from Airborne LiDAR Data using Scene Context", IEEE, pp. 9-16, (2012).

15. F.Samadzadegan, M.Haahn and S.Saeedi, "Position estimation of aerial vehicle based on a vision aided navigation system", Proceedings of Visualization and Exploration of Geospatial dataStuttgart, (2007).

16. David Barry Hester, Halil I. Cakir, Stacy A.C.Nelson and Siamak Khorram, Per-pixel Classification of High spatial resolution satellite imagery for Urban land cover mapping, Photogrammetric Engineering \& Remote Sensing, 74, pp. 463-471, (2008). 
17. P.Sathya and L.Malathi, Classification and Segmentation in Satellite imagery using Back propagation Algorithm of ANN and K-Means Algorithm, International Journal of Machine Learning and Computing, pp. 422-426, (2011).

18. Yoshua Bengio, Learning Deep Architectures for AI, Foundations and Trends in Machine Learning, pp.1-127, (2009).

19. G Hinton and S Osindero, A fast learning algorithm for deep belief nets, Neural computation, pp. 1527-1554, (2006).

20. A Krizhevsky, I Sutskever and G. Hinton, Imagenet classification with deep convolutional neural networks, Advances in neural information processing systems, pp. 1097-1105 (2012).

21. D.I.F Grimes: Optimal areal Rainfall estimation using Rain gauge data and Satellite data, Journal of Hydrology, 222, pp. 93-108, (1999).

22. Farid Melgani and Sebastiano B. Serpico, A statistical approach to the fusion of spectral and spatio-temporal contextual information for the classification of remote sensing images, Pattern Recognition Letters, 23, pp.1053-1061, (2002).

23. Susan Malaso Kotikot and Simon M. Onywere, Application of GIS and Remote sensing techniques in frost risk mapping for mitigating agricultural losses in the aberdare ecosystem, kenya, Geocarto International, 30, (2015).

24. Liu Haijiang, Zhou Chenghu, Cheng Weiming, Long En and Li Rui, Monitoring sandy desertification of Otindag Sandy Land based on multi-date remote sensing images, Acta Ecologica Sinica, 28, 2008.

25. H.S.Lim, M.Z.MatJafri, K.Abdullah, N.Mohd. Saleh and C.J.Wong, Extracting Spatial Data from Satellite Sensor to support Air Pollution Determinant using Remote Sensing Technique, IEEE, pp. 4302-4305, (2007).

26. J. Tian and D. M. Chen, Evaluating Satellite-Based Measurements for Mapping Air Quality in Ontario, Canada, Journal of Environmental Informatics,(2007).

27. Francis Padula, Julia MacDonough, Dan Bondy and Monica Cook, Automated Supervised Land use classification and Change detection: An image fusion based approach, IEEE, , pp.335-338, (2010).

28. Brian P Salmon, Jan Corne Olivier, Konrad J.Wessels, Waldo Kleynhans, Frans van den Bergh and Karen C. Steenkamp, Unsupervised Land cover change Detection: Meaningful Sequential Time Series Analysis, IEEE Journal of Selected Topics in Applied Earth Observation and Remote Sensing, 4, pp. 327-335, (2011).

29. Deepti Sharma, Darshan Singh and D.G.Kaskaoutis, Impact of two dust storms on aerosol characteristics and radiative forcing over Patiala, Northwestern India, Advances in Meteorology, (2012).

30. Biswadip Gharai, Subin Jose and D.V.Mahalakshmi, Monitoring intense dust storms over the Indian region using satellite data - a case study, International Journal of Remote Sensing, 34, pp. 70387048,(2013).

31. Abishek Kumar, Kumar A, Ranjan S and Kumar S, A Rainfall Prediction model using artificial neural network, IEEE, pp. 82-87, (2012).

32. M.Egmont-Petersen, D.de Ridder and H. Handels, Image processing with neural networks-a review, Pattern Recognition, 35, pp. 2279-2301, (2002).

33. Giorgio Giacinto, Fabio Roli, Lorenzo Bruzzone, Combination of neural and statistical algorithms for supervised classification of remote-sensing images, Pattern Recognition Letters, 21, pp. 385397, (2000).

34. L.Castellana, A.D Addabbo and G.Pasquariello, A composed supervised/unsupervised approach to improve change detection from remote sensing, Pattern Recognition Letters, 28, pp. 405-413, (2007).

35. Pabitra Mitra, B.Uma Shankar and Sankar K. Pal, Segmentation of multispectral remote sensing images using active support vector machines, Pattern Recognition Letters, 25, pp. 1067-1074, (2004).

36. Qihao Weng, Dengsheng Lu and Jacquelyn Schubring, Estimation of land surface temperature-vegetation abundance relationship for urban heat island studies, Remote sensing of Environment, 89, pp. 467-483, (2004).

37. Milap Punia, P.K.Joshi and M.C.Porwal, Decision tree classification of land use land cover for Delhi, India using IRS-P6 AWIFS data, Expert systems with Applications, (2010).

38. Zhang Yang, Fu-Lai Chung and Wang Shitong, Robust Fuzzy Clustering-based image segmentation, Applied Soft Computing, 9, pp. 80-84, (2009).

39. Mohamad Awad, An Unsupervised Artificial Neural Network Method for Satellite Image Segmentation, International Arab Journal of Information Technology,7, pp. 199-205, (2010).
40. Ashish Ghosh, Niladri Shekhar Mishra and Susmita Ghosh, Fuzzy Clustering algorithms for unsupervised change detection in remote sensing images, Information Sciences, 181, pp. 699-715, (2011).

41. Xiang-Yang Wang, Xian-Jin Zhang, Hong-Ying Yang and Juan Bu, A pixel-based color image segmentation using support vector machine and fuzzy C-means, Neural Networks, 33, pp. 148-159, (2012).

42. Kazim Hanbay and M.Fatih Talu, Segmentation of SAR images using improce artificial bee colony algorithm and neutrosophic set, Applied Soft Computing, 21, pp. 433-443, (2014).

43. A.K. Bhandari, A.Kumar and G.K.Singh, Modified Artificial Bee colony based computationally efficient multilevel thresholding for satellite image segmentation using Kapur's, Otsu and Tsallis functions, Expert Systems with Applications, 42, pp. 1573-1601, (2015).

44. Yangyang Li, Shixia Feng, Xiangrong Zhang and Licheng Jiao, SAR image segmentation based on quantum inspired multiobjective evolutionary clustering algorithm, Information Processing Letters, 114, pp. 287-293, (2014).

45. Jose M. Pena, Object based image classification of Summer crops with Machine Learning Methods, Remote Sensing, pp. 5019-5041, (2014).

46. T.Blaschke, Object based image analysis for remote sensing, ISPRS Journal of Photogrammetry and Remote Sensing, 65, pp. 2$16,(2010)$.

47. G.J. Hay, G. Castilla, M.A. Wulder and J.R. Ruiz, An automated object-based approach for the multiscale image segmentation of forest scenes, International Journal of Applied Earth Observation and Geoinformation, 7, pp. 339-359, (2005).

48. Muhammad Shahbaz, Aziz Guergachi, Aneela Noreen, and Muhamad Shaheen, Classification by Object Recognition in Satellite images by using Data mining, Proceedings of the World Congress on Engineering, (2012).

49. Rutherford V. Platt and L. Rapoza, An Evaluation of Object oriented paradigm of Land use/ land cover classification, The Professional Geographer, 60, (2008).

50. Petra Helmholz, Franz Rottensteiner and Christian Heipke, Semiautomatic verification of cropland and grassland using very high resolution mono-temporal satellite images, ISPRS Journal of Photogrammetry and Remote Sensing, 97, pp. 204-218, (2014).

51. M. Turker, E. Sumer, Building-based damage detection due to earthquake using the watershed segmentation of the post-event aerial images, International Journal of Remote Sensing, 29, pp. 3073-3089, (2008).

52. D.H. al Khudairy, I. Caravaggi and S. Glada, Structural damage assessments from Ikonos data using change detection, objectoriented segmentation, and classification techniques, Photogrammetric Engineering \& Remote Sensing, 71, pp. 825-837, (2005).

53. L. Monika Moskal, Diane M. Styers and Meghan Halabisky, Monitoring Urban Tree Cover Using Object-Based Image Analysis and Public Domain Remotely Sensed Data, Remote Sensing, 3, pp. 2243-2262, (2011). 